electricity. In the model atom proposed by Sir J. J. Thomson the electrons were supposed to be embedded in a sphere of positive electricity of about the dimension of the atom as ordinarily understood. Experiments on the scattering of $\alpha$-particles through large angles as the result of a single collision with a heavy atom showed that this type of atom was not capable of accounting for the facts unless the positive sphere was much concentrated. This led to the nucleus atom of Rutherford, where the positive charge and also the mass of the atom are supposed to be concentrated on a nucleus of minute dimensions. The nucleus is surrounded at a distance by a distribution of negative electrons to make it electrically neutral. The distribution of the external electrons on which the ordinary physical and chemical properties of the atom depend is almost entirely governed by the magnitude of the positive charge. The experiments by Marsden and Geiger on the scattering of the $\alpha$-particles, and also on the scattering of $\mathrm{X}$-rays by Barkla, show that the resultant units of charge on the nucleus of an element is about equal to its atomic number when arranged in order of increasing atomic weight. Strong proof of the correctness of this point of view has been given by the work of Moseley on the X-ray spectra of the elements, for he has shown that the properties of an element are defined by a whole number which changes by unity in passing from one element to the next. It is believed that the lightest element, hydrogen, has a nuclear charge of one, helium of two, lithium of three, up to the heaviest element, uranium, of charge 92.

Radioactive evidence indicates that the nucleus contains both positively charged masses and negative electrons, the positive charge being in excess. Apart from the difficulty on the ordinary laws of electric forces of explaining why the nucleus holds together, there is a fundamental difficulty of accounting for the stability of the external electrons on the ordinary laws of dynamics. To overcome this difficulty, Bohr has applied the quantum theory to define the position of the electrons and to account for the spectra of the lighter atoms and has made suggestions of the structure of the simpler atoms and molecules. Space does not allow me to discuss the important developments that have followed from Bohr's theory by the work of Sommerfeld, Epstein, and others. The generalised theory has proved very fruitful in accounting in a formal way for many of the finer details of spectra, notably the doubling of the lines in the hydrogen spectrum and the explanation of the complex details of the Stark and Zeeman effects. In these theories of Bohr and his followers it is assumed that the electrons are in periodic orbital motion round the nucleus, and that radiation only arises when the orbit of the electron is disturbed in a certain way. Recently Langmuir, from a consideration of the general physical and chemical properties of the elements, has devised types of atom in which the electrons are more or less fixed in position relatively to the nucleus like the atoms of matter in a crystal. It appears necessary, in Langmuir's theory, to suppose that electrons, in addition to their electrical charges, are endowed with the properties of a magnetic doublet, so that at a certain distance the forces of attraction and repulsion between two electrons counterbalance one another.

The whole question of the possible arrangements and motion of the external electrons in an atom or molecule still remains a matter of much doubt and speculation. While there are strong indications that the conception of the nucleus atom is in the main correct, we are still very uncertain of the laws controlling the position of the external electrons on which the ordinary physical and chemical properties depend. The study of the light spectra and also of the X-ray spectra already promise to throw new light on this very difficult but fundamental problem.

From the above hurried survey of the progress of atomic physics, it will be seen that the investigations of the past twenty-five years have deait mainly with three great outstanding problems, viz., the nature of electricity, the structure of the atom, and the nature of radiation. While great additions have been made to our knowledge of these questions leading to a much wider outlook, we cannot but recognise that much still remains to be done before we are certain that we are building on a firm foundation for the future. Notwithstanding the prolonged halt during the war, the scientific outlook is one of good augury for the immediate future, and there is every prospect that the vigorous attack on these outstanding problems will be continued.

\title{
ATOMS AND MOLECULES.
}

\section{By Prof. Frederick Soddy, F.R.S.}

[ $\mathrm{T}$ may be doubted whether, fifty years ago, chemists and physicists believed very deeply in the actual reality of the molecules and atoms, which they used as convenient and simplifying conceptions to interpret the behaviour of matter. The half-century, indeed, has not passed without strong protest from the thermodynamical school of physical chemistry that the science should be NO. 26 IO, VOL. IO4] so wedded to pure hypotheses and unverifiable assumptions, then, apparently, for ever beyond the power of being actually apprehended and demonstrated. That the modern student of physical science believes in the reality of the existence of his atoms and molecules, as much as he does in that of chairs, tables, and lampposts, probably sufficiently epitomises one of the 
most striking features of the change of outlook since NATURE made its first appearance in 1869. Vague ideas of their actual individual mass, size, shape, and constitution have been or are being replaced more and more by exact quantitative knowledge, which invites our literal acceptance and grows in fruitfulness the more implicitly it is used as the basis for further investigations.

But the latter half of the period under review witnessed an even greater change of outlook. The atom, since the discovery of radio-activity in 1896, has ceased to be the smallest coin of the realm of material change. The farthings of 1869 have proved to resemble roool. notes, and the potentialities of the world in consequence have been multiplied a million times. The change of the single atom of matter is well within the range of direct perception by the senses, and, stranger still, the change reveals that, under the image and superscription of the same Cæsar, coins of different mass and mintage have been circulating unsuspected in the chemist's currency.

As regards the physical reality of molecules, by no means the least important factor contributing to the result has been the recognition that, if the molecules were not the smallest parts of matter capable of free independent existence and motion, heat would not be the final permanent form which all kinetic energy liberated in the world assumes. The limit that fixes the physical sub-division of matter limits also the sub-division of motion. Though in the real world of matter in bulk, as contrasted with the ideal fictions of mathematics, friction and imperfect elasticity quickly reduce all moving masses to apparent rest, that "rest" is the perpetual heat motion of the molecules, which, literally and necessarily, must be perfectly frictionless and elastic because they are the smallest particles capable of free independent motion, and no smaller particles exist among which their motion can be further distributed.

Moreover, in accordance with the law of equipartition of energy, all molecules at the same temperature, whatever their mass, become, in consequence of their ceaseless mutual collisions, possessed of the same average amount of kinetic energy, and, therefore, of a velocity of translation inversely proportional to the square-root of their mass. This serves to clarify the conception of the real molecule from misnomers still unthinkingly retained.

For example, it is a pure survival of past confusion to speak of the molecule of a crystalline solid, if not of any solid, for in such the smallest parts are not free to move, but are anchored in fixed, unchanging positions in the crystal spacelattice, as the resolution of X-rays by the crystal structure has shown. It is, similarly, always a pure misnomer to give the name "molecule" to the least number of atoms which represent the chemical composition and properties of a substance, in the absence of experimental knowledge of the molecular magnitude, and therefore of any knowledge as to whether such a particle really NO, 26IO, VOL. IO4] exists in a form capable of free independent movement.

Cleared of these ambiguities, the conception of the individual molecule has become very real. We have been led by Perrin, and the mathematical physicists who paved the way for his experimental work, to recognise the Brownian movement as but one aspect of the perpetual motion of the molecules, which, though invisible to the naked eye, becomes swift and ceaseless for particles even of the scale of minuteness resolved by the microscope, and we can extrapolate with assurance to the minuter world which science had long before visualised by faith.

Or, again, we may follow Langmuir, with none of the feeling of hesitancy and diffidence that would have held back an earlier generation, into the explanation of catalysis, adsorption, and allied phenomena, as caused by surface layers of molecules "one molecule thick." Nor do we consider it fanciful to explain the spreading of animal and vegetable oils upon water and the non-spreading of mineral oils, as due to the attempt, in the first case, of the one end, the soluble glycerine ester end, of the rod-like molecule to dissolve in the water, and the refusal of the other end, the insoluble, hydro-carbon, or oily end, to do so. Wherefore the molecules of such oils stand up on end and cover the surface with a one-molecule thick layer of the oily ends of the molecules, whereas the mineral oils, with molecules oily at both ends, do not spread! Real in one sense as the structural formulæ of organic compounds have been for many decades, an earlier generation would scarcely have thought of this.

The discovery that the X-rays are of a character identical with light, but of wave-length of the order of one ten-thousandth of that of light of the visible spectrum, has made the structure of crystalline solids as open to direct examination as the ten-thousand-fold coarser structure of the Rowland grating, ruled by the dividing engine, is by means of ordinary light. In this way many of the space-lattices hitherto arrived at only by the aid of the second-sight of the mathematical crystallographer have been tested and found real.

Since the explanation by Le $\mathrm{Bel}$ and van't Hoff of optical isomerism as due to structural differences of the arrangement of the atoms in the molecule, of the kind that exist between an asymmetrical object and its mirror-image, and therefore only capable of representation in space of three dimensions, chemists have, not without reproach, used model carbon atoms in building up the structure of organic compounds, and have found them capable of accounting, for example, in cyclic structures, for many of the properties of these compounds far removed from the field of optical activity. That the real carbon atom should possess any resemblance to these little wooden balls bearing four spokes radiating symmetrically from the centre may have appeared to many too crude a conception for literal belief. Yet when the character of the space-lattice of the diamond crystal 
was elucidated by means of the X-rays, these very models were used to represent it-a striking proof, surely, of the basis of physical reality underlying the conceptions of stereo-chemistry.

But these triumphant vindications of what only a generation ago were described as purely hypothetical and unverifiable conceptions have been to some extent overshadowed and eclipsed by the startling progress made since the discovery of radio-activity in 1896 and its almost immediate interpretation as due to the explosive disintegration of the atoms of the radio-elements. This subject is being treated by Sir Ernest Rutherford in another article, and need be only briefly alluded to here. The change is attended by the liberation of energy a million times greater than is liberated in any previously known change of matter, and so it has come about, as for example in the spinthariscope, that the effect of each individual atom disintegrating can be perceived by the senses. The counting of the number of atoms disintegrating per minute has become one of the regularly used methods of investigation, whereas it requires, at least, some 25,000 times as many atoms as there are people alive in the world before an element can be detected by the spectroscope. The condensation of moisture on the columns of ions, lying in the tracks of the fragments of the atom after its explosion-both of the $\alpha$ - and $\beta$-particles, which may be likened to projectiles fired from a gun, and of the recoiling residue of the atom or gun itself-has in the hands of C. T. R. Wilson enabled the individual atomic explosions to be photographed. These permanent records, of extraordinary interest and value as they are as confirmatory evidence, yet revealed nothing new. Every detail of the whole phenomenon had been correctly comprehended and established without such direct aid. In particular, the photographs show well the almost rectilinear flight of the $a$-particle through the myriads of gas atoms in their path, and their rare and occasional wide-angle deviation when perchance they pass near enough to the heart of the atoms penetrated, which is the experimental basis for the present provisional representation of the internal structure of atoms.

The atom is regarded now as a solar system, but the massive central sun, comprising all but a negligible fraction of the whole mass, is an exceedingly minute positively charged nucleus, attended by numerous rings or shells of the almost mass-less electrons. In spite of its relatively great mass, the nucleus is so minute that the chance of an $\alpha$-particle-which itself is the nucleus of a helium atom-in its passage through the atom approaching or colliding with the central nucleus, is exceedingly small. Mass and radioactivity alone seem to depend directly upon this hitherto unsuspected and all-important nucleus. The chemical and physical properties, including the light spectrum, are governed probably by the outermost shell or ring of valency electrons, which alone are variable in number. The coming and going of these seem to constitute chemical NO. $26 \mathrm{IO}, \mathrm{VOL} . \mathrm{IO} 4]$ change and to give rise to ordinary light radiation. Barkla's various series of $\mathrm{X}$-rays characteristic of each element probably originate in the successive completed rings or shells of electrons surrounding the nucleus.

All the properties of the atom, practically, save mass and radio-activity, depend solely upon the numerical value of the positive charge of the nucleus, which is equal to the number of the surrounding negative electrons. This number, which is known as the atomic number, increases unit by unit in passing from one place of the periodic table to the next. From numerical relationships between the wave-lengths of the characteristic X-rays, Moseley was able to determine or infer this atomic number for all the elements. So he called the roll of the elements for the first time and found between hydrogen, the first, and uranium, the last and ninety-second element in the table, only five still missing.

In the course of successive radio-active changes the radio-element expels from its nucleus an $\alpha-$ or $\beta$-particle, so losing two positive charges, or, relatively, gaining one, and shifting back two places or moving forward one in the periodic table. The expulsion of one $\boldsymbol{\alpha}$ - and two $\beta$-particles produces an isotope of the parent, chemically and spectroscopically identical with it, but of atomic mass four units less. The ultimate products of uranium and thorium have been identified as isotopes of lead of atomic mass 206 and 208 respectively, and this has been confirmed by an examination of the atomic weight of the lead derived from uranium and thorium minerals. Of all strange consequences of the atom changing, this is perhaps the most subtle and hitherto unsuspected, for now nothing is more certain than that the analysis of matter into chemical elements depends on a superficial identity of the outer shell of the atom, and that the same type of outer shell may contain internal nuclei of different mass and different constitution.

Naturally, the many, at first separate and independent, lines of evidence which have led to the present results cannot all be even mentioned in an article of this length. The significant fact is that all the new and powerful methods of attack developed by physics and chemistry during the last quarter of a century are converging successfully on the problem of the internal constitution of the atoms. The prospects of successful accomplishment of artificial transmutation brighten almost daily. The ancients seem to have had something more than an inkling that the accomplishment of transmutation would confer upon men powers hitherto the prerogative of the gods. But now we know definitely that the material aspect of transmutation would be of small importance in comparison with the control over the inexhaustible stores of internal atomic energy to which its successful accomplishment would inevitably lead. It has become a problem, no longer redolent of the evil associations of the age of alchemy, but one big with the promise of a veritable physical renaissance of the whole world. 
Even in the present year a furtiner significant advance in this direction has been made. For it appears, from the latest results of Sir Ernest Rutherford on the passage of $\alpha$-particles through nitrogen, as though the nuclei of an exceedingly minute proportion of the nitrogen atoms struck by the $a$-particle were shattered by the collision. If this is so, artificial transmutation on an infinitesimal scale has already been accomplished, though, it is true, only by the aid of a previous natural transmutation, still impossible artificially to imitate.

\section{IONISATION OF GASES.}

\section{By Prof. J. S. Townsend, F.R.S.}

$\mathrm{D}^{\mathrm{U}}$ URING the last fifty years many physicists have been occupied in studying problems connected with electric currents in gases. The earlier work was principally confined to experimental investigations of the general outlines of the phenomena which occur in discharges obtained with high potentials. The large number of complicated and surprising properties of gases which were thus discovered naturally attracted much attention, and it is very interesting to read the accounts of the first experiments of the discharges in air and through vacuum tubes which were written before any special investigations of the theory of the conductivity were undertaken.

It would clearly have been extremely difficult to obtain from these experiments any general theory of electricity to explain what was taking place, as such a large number of different phenomena seemed to occur simultaneously. From the first, some physicists maintained that the currents through gases were carried by means of ions, as in liquids, although there were peculiar differences between the two cases, and it was not evident why under a given force a gas might act either as an insulator or as a conductor.

The greatest success in advancing the theory of electricity was obtained from careful studies of the discharges at very low pressures. In this direction some remarkable experiments on the cathode rays were made by Hittorf in 1869 . He found that the rays travelled in straight lines from the cathode; when they fell on glass they caused the surface to fluoresce, and an obstacle in the path of the rays cast a shadow on the glass. $\mathrm{He}$ also found that the rays were deflected by a magnet into circles, or more generally into spirals, which were described in the direction which would be taken by negatively charged particles moving from the cathode. Notwithstanding these results, and further experiments made by Crookes, the projected particle theory of the rays was not at first universally accepted, and some physicists maintained that the rays were an undulatory motion of the ether. This question was decided by Perrin in 1895 . He showed by direct experiment that the rays carried a negative charge, but thus far the origin of the rays, their velocity, and the mass and charge of each particle were unknown.

The question of the ratio of the charge to the mass was studied by Schuster in r890, and he concluded that in gases it was of the same order as in liquids, but for negative ions it was larger NO. 26 IO, VOL. IO4] than for positive. This was the first indication of the characteristic difference between positive and negative ions in gases.

A direct method of finding the ratio of the charge $e$ to the mass $m$ of the cathode particles, and the velocity of the particles, was devised by Wiechert, and in 1897 he described the experiments which showed that in some cases the velocity of the rays was about one-tenth of the velocity of light, and that the ratio $e / m$ for the cathode rays was between 4000 and 2000 times as great as the corresponding quantity for a hydrogen atom. Thus, assuming the charges to be the same in the two cases, the experiments showed that the mass of a cathode particle is very small compared with the mass of an atom of hydrogen. This small cathode particle has been called the electron. Further experiments show that currents of negative electricity obtained from metal surfaces by other methods also consist of streams of electrons. Thus Sir J. J. Thomson investigated the charged particles set free from hot wires or from a metallic surface by the action of ultraviolet light, and found that in both cases the ratio $e / m$ was the same as for cathode rays. The values of $e / m$ afterwards found by various methods show that the ratio of the mass of the electron to the mass of an atom of hydrogen is I : 1830 . This value of $e / m$ is constant provided the velocity is small compared with the velocity of light; but with velocities of this order the effective mass of the electron increases, and Kaufmann found that the value of $e / m$ diminishes in accordance with Lorentz's theory as the velocity approaches the velocity of light.

During the earlier part of this period some investigations were made of the currents that can be obtained with forces smaller than those required to produce discharges. The positive and negative ions produced in air at atmospheric pressure at the surface of incandescent metals, the conductivity of flames, and the charges obtained in newly prepared gases, or by bubbling air through water, were examined. In these cases the mass associated with the ions is comparatively large, and varies rather irregularly over wide ranges, so that it was difficult to formulate precise theories from the results of the experiments. These large ions have the property of condensing water vapour, and in a moist atmosphere small drops are easily obtained which form a visible cloud. This phenomenon led to the method of estimating the charge on each particle. The number of 